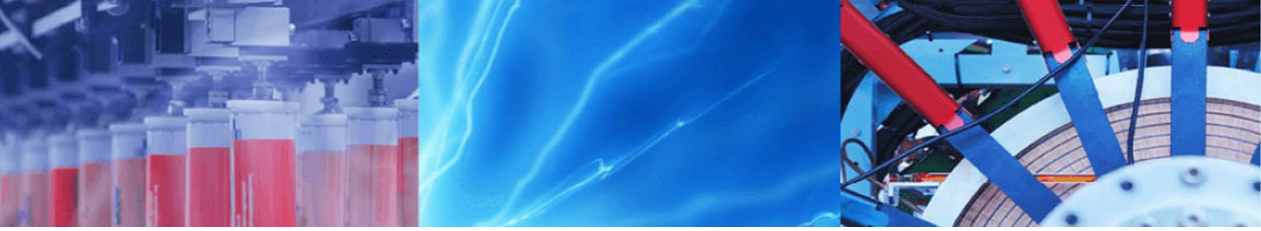

Research Article

\title{
High filtration efficiency fluffy material: nano-fiber constructing gradient structure on recycled curved PET micro-fibers web
}

\author{
Lingli Deng ${ }^{1} \cdot$ Peng Zhang ${ }^{2} \cdot$ Wentao Zhang ${ }^{1} \cdot$ Ruitian $\mathrm{Zhu}^{3} \cdot$ Yurong Yan ${ }^{1}$ (D)
}

(c) Springer Nature Switzerland AG 2019

\begin{abstract}
High efficiency filtration materials focus on electreted melt-blown polypropylene nonwoven and nano-scale fibers membrane in industry. However, the charge decay and the balance of efficiency and resistance are still facing challenges on research and practical applications. In this research, combined with traditional electrospinning and carding process, a simple and new fluffy gradient filtration material was designed by constructing nano-scale poly(lactic acid) (nano-PLA) fiber membranes on recycled three-dimensional curved poly(ethylene terephthalate) (R-PET) micro-fibers webs. Influences of basis weights of nano-PLA membranes and curved types of R-PET fibers on the filtration performance and the dust-holding capacity of R-PET/nano-PLA mats were investigated. Besides, the filtration mechanism of R-PET/nano-PLA mats was systemically studied. Results show that R-PET with zigzag curve (R/Z-PET) possesses the highest filtration efficiency of $99.992 \%$ comparing with $99.517 \%$ of linear R-PET, the pressure drop fall to $201.11 \mathrm{~Pa}$, and a quality factor level up from 0.024 to $0.047 \mathrm{~Pa}^{-1}$. The pressure raising rate of the R/Z-PET/nano-PLA mat is the slowest. R/Z-PET fibers with a folding angle of $90^{\circ}$ benefit to decrease the friction between fiber and particles when the location angle of zigzag fiber is $45^{\circ}$ along the airflow direction, which decrease the pressure drop at high filtration efficiency. Surface residual charges contribute some effect on the overall filtration performance of the R-PET/nano-PLA mat. A new way of simple curved micro-PET fiber combining with nano-scale fibers provides an industrial available path to mass production of high efficiency filtration material serving for air pollution control without electret process.
\end{abstract}

Keywords Poly(lactic acid) · Zigzag curve · Fluffy structure $\cdot$ Recycled poly(ethylene terephthalate) $\cdot$ Air filtration

\section{Introduction}

Environmental pollution is becoming one of the most serious problems facing mankind today. Air pollution control has aroused people's fervent concern owing to the worsening smog problem [1, 2]. Conventional fibrous filtration materials including textiles, melt-blown nonwovens, needle-punched nonwovens and spun-bond nonwovens cannot meet the requirements of high filtration efficiency (FE) and energy saving in filtration industry
[3-5]. Polypropylene (PP) melt-blown electret material has provided a new innovation method of high FE combining with low pressure drop $(\Delta P)$ due to abundant charges on the surface of fibers [6-8]. However, the charges decay with time in service or storage conditions, resulting in the reduction of FE [9].

Electrospun nanofiber membranes possess many advantages such as interconnected open pores, small pore size, high porosity and large surface-to-volume ratio, which make them promising candidates for air filtration. Al-Attabi [10],

Electronic supplementary material The online version of this article (https://doi.org/10.1007/s42452-019-0209-x) contains supplementary material, which is available to authorized users.

\footnotetext{
$\triangle$ Yurong Yan, yryan@scut.edu.cn | ${ }^{1}$ School of Materials Science and Engineering, South China University of Technology, Guangzhou 510640, China. ${ }^{2}$ Guangzhou Fiber Product Testing and Research Institute, Guangzhou 511447, China. ${ }^{3}$ Guangzhou Inspection Testing and Certification Group Co., Ltd., Guangzhou 511447, China.
} 
Wang [11] and Zhang [12] found that nanofiber membranes have superior FE owing to the nano-scale fiber diameter and small pore size, but they also exhibit high airflow resistance due to the compact structure [13].

In order to decrease airflow resistance while keep the high $\mathrm{FE}$, an ideal filtration material is elaborately designed with a kind of gradient structure which provides an effective surface area inside filtration materials [14]. On the other hand, the structure facilitates the pressure gradient, improves FE and reduces the airflow resistance [15-20]. Micro/nano-fibers, nanoparticles and multi-layer hybrid structure are used for the designing of the gradient structure. Cheng et al. [21] introduced a PAN/PVA multi-level structure filtration material via multi-jet electrospinning process, and its FE was up to $99.996 \%$ and $\triangle \mathrm{P}$ reached $418 \mathrm{~Pa}$ for the testing media particles size of $300 \mathrm{~nm}$.

Improved works have been reported by Ding et al. [22, 23], who designed a fluffy material containing micro/ nanofibers or multi-layer hybrid membranes using electrospinning technique and achieved the highest FE more than $99.99 \%$. A special fluffy structure composed of larger cavities inside the hybrid filtration materials reduces the friction between the airflow and fibers greatly, resulting in the extremely low $\Delta \mathrm{P}$ less than $110 \mathrm{~Pa}$ [24].

When raw materials using in air purification are marked with GREEN, they are always in a favorable pole in the selection list. Post-used polymer materials are one of them, and have been studied and evaluated for decades [25-28]. Recycled polyethylene terephthalate (R-PET) fiber can be made from post-used bottles and clothing. More significantly, fibers with unique curved structure can be easily produced thanks to the modern spinning technology, which reduce the cost of air filtration media [28-30]. The curved R-PET fibers exhibit a fluffy porous threedimensional (3D) structure inside webs. However, special designed curved R-PET fiber matrix combining with nanofiber fluffy materials is rarely reported.

In this research, we used micro R-PET fibers with different curved shape as the matrix fiber web, nano-scale biodegradable electrospun poly(lactic acid) (PLA) [31] membrane as the fluffy functional filtration layer, and designed a series of fluffy filtration materials. The filtration performance and mechanism of micro/nanofiber gradient structure filtration material controlled by different curved fibers were systemically studied and analyzed.

\section{Experimental section}

\subsection{Materials}

PLA $\left(M_{w}=1.6 \times 10^{5} \mathrm{~g} \mathrm{~mol}^{-1}, 6202 \mathrm{D}\right)$ was produced by Natureworks LLC, USA. N, N-dimethylformamide (DMF, analytical grade) and dichloromethane (DCM, analytical grade) were purchased from Shanghai Chemical Reagents Co. Ltd., China.

R-PET webs were provided by Guangdong Jiangmen Yuexin Chemical Fiber Co. Ltd. The classification of R-PET substrates is shown in Supporting Information (Table S1). Here, $\mathrm{R}_{1.5} / \mathrm{N}$-PET and $\mathrm{R}_{2.5} / \mathrm{N}$-PET mean R-PET fibers with linear shaped and the fiber fineness are 1.5 denier and 2.5 denier, respectively. R/S-PET means R-PET fiber of 7 denier with S-shaped curve and R/Z-PET is R-PET of 7 denier with zigzag-shaped curve.

\subsection{Preparation of nano-PLA/R-PET mats}

PLA was dissolved in a mixed solvent of DCM and DMF at a volume ratio of 4 to 1 using Magnetic Stirring Setup HJ-4A (Changzhou Guohua Electric Appliance Co. Ltd., China) at $25^{\circ} \mathrm{C}$. The concentrations of PLA solutions were $8,10,12$ and $14 \mathrm{wt} \%$, respectively.

The electrospinning conditions to obtain various nanofibers membranes were shown in Support Information (Table S2). The nano-PLA membranes were prepared via ESF-Y1 Electrospinning Instrument [32] deposited on aluminum foil substrates or different R-PET webs.

\subsection{Characterization}

The viscosity of PLA spinning solutions was measured using a DV2TLVTJO rotational viscometer (Brookfield, USA). The conductivities of PLA solutions were tested on a CON510 Conductivity Meter (Singapore EUTECH Company, Singapore). The surface tensions of solutions were tested using the maximum bubble method [33]. For all testing, the final results were average values based on five parallel results and error analyses were considered.

The morphology of the fibers was observed using Quanta 200 Environmental Scanning Electron Microscope (Netherlands FEl Co., Netherlands) and Axioskop 40 POL Polarizing Microscope (Carl Zeiss, Germany). The fiber diameters were analyzed with Digimizer software.

Basis weights of PLA electrospun membrane on R-PET substances were measured according to ISO 9073-1 and the deviations were controlled.

The permeability of samples was characterized using FX3300 Fabric Breathability Tester (Switzerland TEXTEST, Switzerland). The tested sample area was $20 \mathrm{~cm}^{2}$ and an applied pressure was $200 \mathrm{~Pa}$. All result values were average of three parallel results.

The pore sizes and their distributions of samples were analyzed on a CFP-1200AP Capillary Flow Porometer (PMI Co., USA). 
The surface voltages of filtration materials were measured on a SIMCO FMX-003 Electrostatic Field meter (SIMCO Co., Japan) at standard environment.

The aerosol filtration properties of samples were evaluated using a TSI 8130 Automatic Filtration Material Tester (TSI Co., USA). Sodium chloride $(\mathrm{NaCl})$ aerosol with a mass median diameter of $260 \mathrm{~nm}$ was used. The flow rate was $32 \mathrm{~L} \mathrm{~min}^{-1}$ or the airflow velocity was $5.3 \mathrm{~cm} \mathrm{~s}^{-1}$. The valid testing area of samples was $100 \mathrm{~cm}^{2}$.

\section{Results and discussion}

\subsection{Structure design of the R-PET/nano-PLA mats}

The structure of fluffy filtration materials is presented in Fig. 1. Here, the R-PET/nano-PLA mats are composed of micro-R/Z-PET fiber web and nano-PLA fiber membrane. R/Z-PET web serves as the receiving substrate, and an interpenetrating micro/nanofibers gradient layer is formed between micro-R/Z-PET web and nano-PLA membrane. Curved R-PET and micro/nanofibers layer establish a 3D loosing structure which benefit to high $\mathrm{FE}$ and low $\Delta \mathrm{P}$.

\subsection{Preparation and characterization of the nano-PLA fiber and R-PET/nano-PLA mats}

The morphology and average fiber diameter of electrospun nanofiber are affected by solution viscosity, solution conductivity, applied voltage, distance from tip to collector, surrounding temperature and humidity, and flow rate of solution [34]. An appropriate formation of
Taylor cone is important for the achievement of continuous and stable electrospinning process [15]. The viscosity, conductivity, and surface tension of the PLA solution at various concentrations are shown in Fig. S1 (Supporting Information). The morphologies of nano-PLA fibers are given in Fig. 2.

According to Fig. 2, the morphologies of nano-PLA fibers transform from beads to continuous fibers with the increasing concentration of PLA solutions. High solution viscosity always results in large fiber diameter [35, 36]. The voltage, tip-to-collector distance and spinning rate also influence the morphology of nanofibers (Supporting Information, Figs. S2, S3 and S4). The nano-PLA fiber diameters are $300 \pm 70 \mathrm{~nm}, 740 \pm 200 \mathrm{~nm}, 770 \pm 170 \mathrm{~nm}$, and $1300 \pm 300 \mathrm{~nm}$ with PLA concentrations of $8,10,12$, and $14 \mathrm{wt} \%$, respectively, when the spinning voltage is $15 \mathrm{kV}$, the tip-to-collector distance of $8 \mathrm{~cm}$, and the spinning rate of $3.2 \mathrm{~mL} \mathrm{~h}^{-1}$. The nano-PLA fiber preparing from the PLA solution of $12 \mathrm{wt} \%$ possesses uniform fiber diameter and narrow fiber diameter distribution. Then, the PLA solution of $12 \mathrm{wt} \%$ was used to prepare R-PET/ nano-PLA mats in this research.

To further investigated structure of prepared materials, the pore size distribution of filtration materials was studied. The pore size distribution of R-PET web and R/Z-PET/nano-PLA mat exhibits well-developed peak centering and the pore size range vary from 1.2 to 3.4 and from 1.3 to $5 \mu \mathrm{m}$, respectively (Fig. 3 ). Due to the wide pore size distribution of R/Z-PET web (range from 11.0 to $350.0 \mu \mathrm{m}$ ), the pore size distribution of R/Z-PET/ nano-PLA mat become widening, which are determined by the shapes of the receiving substrate [37].

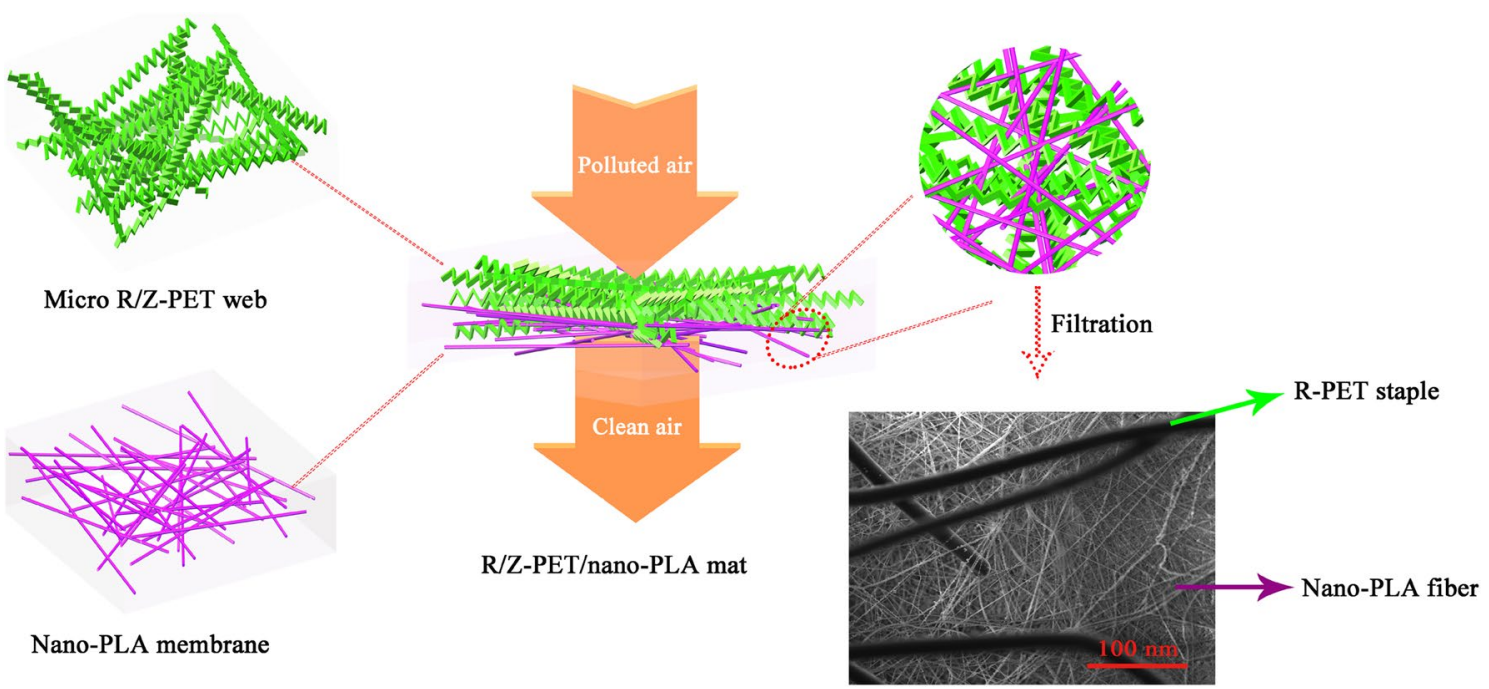

Fig. 1 Structure of 3D R/Z-PET/nano-PLA fluffy filtration material 

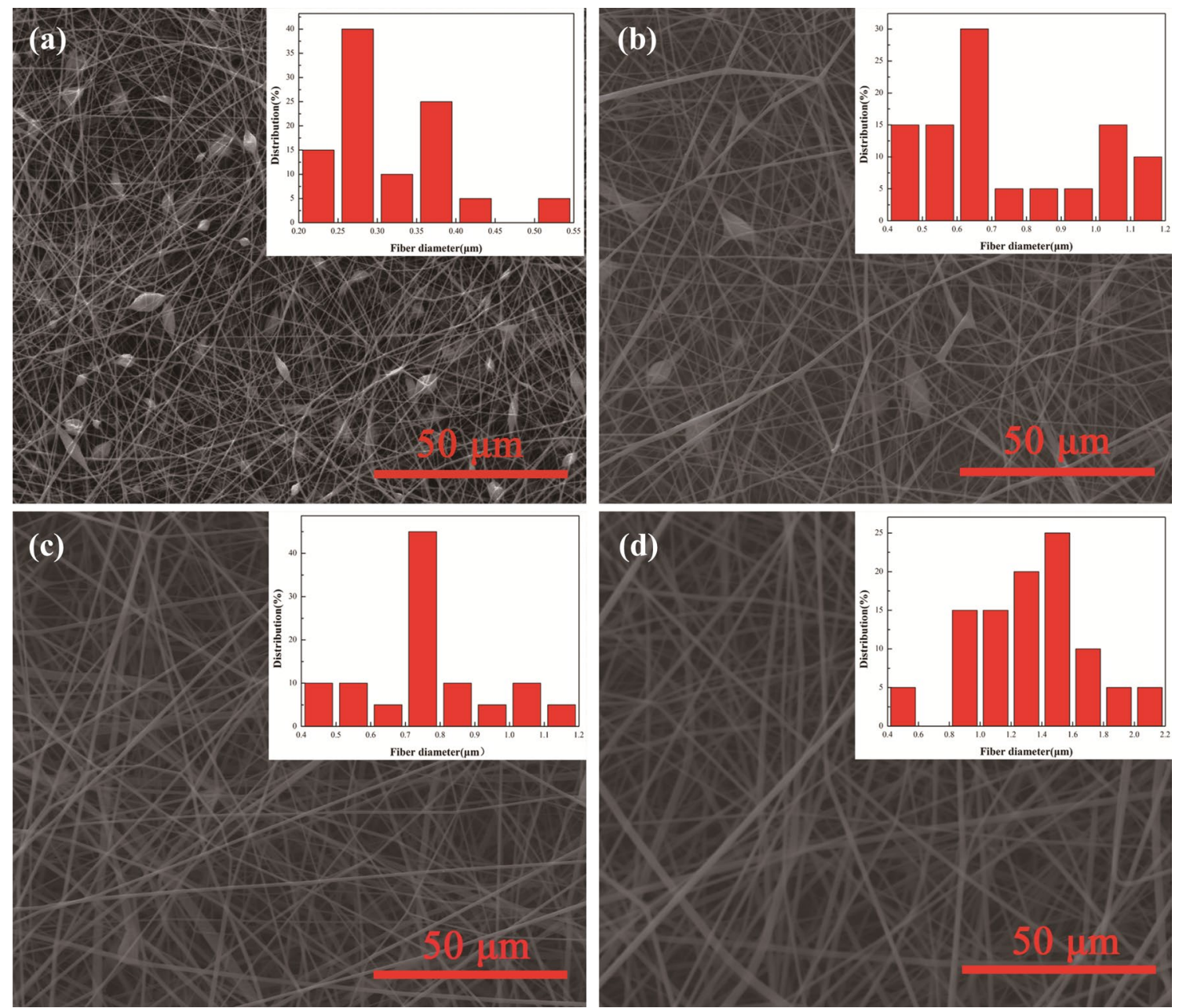

Fig. 2 SEM images of electrospun PLA fibers with PLA concentrations of a 8 wt $\%$, b $10 w t \%, c 12$ wt $\%$, and d 14 wt $\%$, respectively

\subsection{Filtration properties of R-PET/nano-PLA mats with different curved structures of R-PET fibers}

Filters combined with fine fiber always show high FE, but increase in the airflow resistance [38], while the fluffy structure filters can reduce resistance.

As shown in Fig. 4, FE of $\mathrm{R}_{1.5} / \mathrm{N}-\mathrm{PET} /$ nano-PLA, $\mathrm{R}_{2.5} / \mathrm{N}-\mathrm{PET} /$ nano-PLA, R/S-PET/nano-PLA and R/Z-PET/ nano-PLA mats are $99.986,99.517,99.949$ and $99.992 \%$, respectively, and corresponding $\Delta \mathrm{P}$ are $253.33 \mathrm{~Pa}$, 226.11 Pa, 198.27 Pa and 201.11 Pa, respectively. The combined effect of fiber size and shape need to be considered for curved fibers as filtration materials. The fineness of fiber will advantage to $\mathrm{FE}$, while the fiber shape to change the airflow mechanism.

The quality factor (QF) can be used to evaluate the filtration capacity of filtration material by Eq. 1 [16].

$Q F=\frac{-\ln P}{\Delta p}$

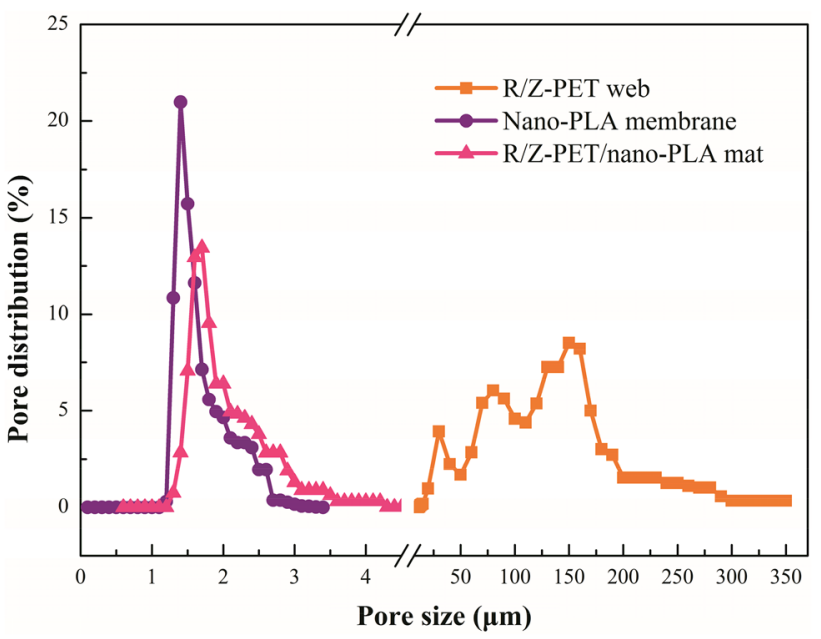

Fig. 3 The pore size distribution curves of R-PET web, nano-PLA membrane and R/Z-PET/nano-PLA mat (the basis weight of nanoPLA membrane is $9 \pm 0.1 \mathrm{~g} \mathrm{~m}^{-2}$ ) 
Fig. $4 \mathrm{FE}$ and $\Delta \mathrm{P}$ of filtration materials changed with fiber fineness and curved shape of R-PET fiber in R-PET/nanoPLA mats (the basis weight of nano-PLA membrane is $9 \pm 0.1 \mathrm{~g} \mathrm{~m}^{-2}$ )

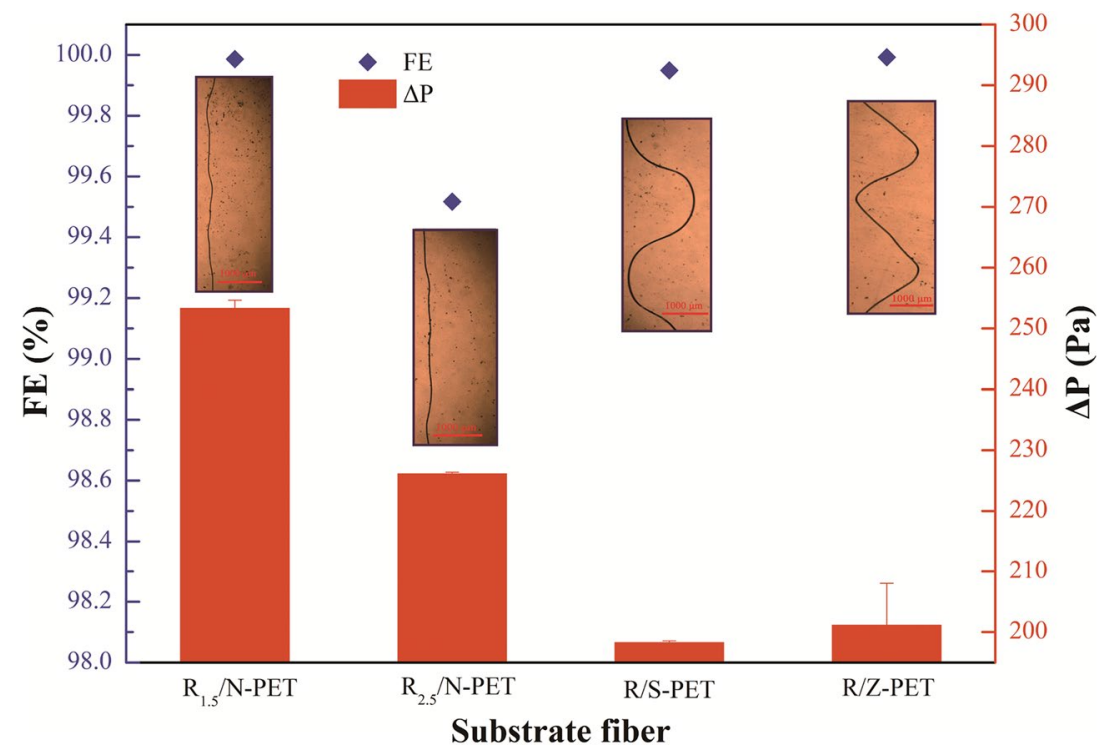

Table 1 QF values of R-PET/nano-PLA mats with different curved structures of R-PET fiber

\begin{tabular}{ll}
\hline Filtration materials & $\mathrm{QF}\left(\mathrm{Pa}^{-1}\right)$ \\
\hline $\mathrm{R}_{1.5} / \mathrm{N}-\mathrm{PET} /$ nano-PLA & 0.035 \\
$\mathrm{R}_{2.5} / \mathrm{N}-\mathrm{PET} /$ nano-PLA & 0.024 \\
$\mathrm{R} / \mathrm{S}-\mathrm{PET} /$ nano-PLA & 0.038 \\
$\mathrm{R} / \mathrm{Z}-\mathrm{PET} /$ nano-PLA & 0.047 \\
\hline
\end{tabular}

where $P$ is the particle penetration (\%) and $\Delta p$ is the pressure drop $(\mathrm{Pa})$.

$\mathrm{QF}$ is a parameter on the energy expenditure in filtration, and influences by particle size and filtration velocity, regardless of thickness. In terms of FE or airflow resistance, a high QF usually shows a superior filtration performance [39]. Here, QF was used to evaluate the influence of curved shapes of R-PET on filtration performance and values are listed in Table 1. The R/Z-PET/nano-PLA mat shows the maximum $\mathrm{QF}$, which means this mat is provided with the optimal comprehensive filtration performance.

\subsection{Filtration properties of R/Z-PET/nano-PLA mats with different basis weight of nano-PLA membranes}

In order to optimize filtration performance of the R/Z$\mathrm{PET} /$ nano-PLA mat, the fluffy filtration materials were fabricated with various basis weight of nano-PLA membranes including $4 \pm 0.2,5 \pm 0.1,6 \pm 0.2,7 \pm 0.2,8 \pm 0.1$ and $9 \pm 0.1 \mathrm{~g} \mathrm{~m}^{-2}$, respectively.

When the basis weight of nano-PLA membrane increased from 4 to $9 \mathrm{~g} \mathrm{~m}^{-2}$, the average pore size of nano-PLA membrane decreases from 2.15 to $1.62 \mu \mathrm{m}$, and R/Z-PET/nano-PLA mat from 3.17 to $1.99 \mu \mathrm{m}$, respectively (Fig. 5), but all are smaller than that of R/Z-PET web $(138.12 \mu \mathrm{m})$. Meanwhile, the breathability of R/Z-PET/ nano-PLA mat decreases from 98.95 to $39.17 \mathrm{~mm} \mathrm{~s}^{-1}$. The increasing basis weight of the nano-PLA membrane enhanced the entanglement percentage between fibers in per unit area [40,41]. At the same basis weight, the average pore size of R/Z-PET/nano-PLA mat decreases comparing with that of the nano-PLA membrane, and this is the result of modification of R/Z-PET web on the pore structure of mat.

As shown in Fig. $6 a$, $b$, the FE and $\triangle \mathrm{P}$ of R/Z-PET/nanoPLA mats elevate with the increasing basis weight of nanoPLA membrane. As the loading time extended, the corresponding $\mathrm{FE}$ and $\triangle \mathrm{P}$ accordingly increase at the same time. FE of R/Z-PET/nano-PLA mat with the low $\left(4 \mathrm{~g} \mathrm{~m}^{-2}\right)$ basis weight of nano-PLA membrane increases rapidly at first, and then undergoes a gradual change after the filtration time exceeds 2 min (Fig. 6a). Rising tendency of FE decreases with weight of nano-PLA membrane increasing. This phenomenon could be essentially ascribed to gradual accumulation of the trapped particles in pore channels inside the filtration material, and step by step, the particles block the channels with increasing filtration loading time, which reduces the pore size inside the filtration material [13]. This tendency is especially obvious for filtration material with a low initial FE value. With the increasing loading time, the following of cake filtration mechanism of the mat results in the increase of $\Delta P$ (Fig. 6b) [42]. As shown in Fig. $6 \mathrm{c}$, the growth rate of $\triangle \mathrm{P}$ of $\mathrm{R} / \mathrm{Z}-\mathrm{PET} /$ nano-PLA mat with increasing basis weight of nano-PLA shows a slow tendency at the beginning and then increase. This result is consistent with the trend of the average pore size of R/Z-PET/nano-PLA mat (Fig. 5). 
Fig. 5 Average pore size of nano-PLA membrane, R/Z-PET/ nano-PLA mat, and breathability of R/Z-PET/nano-PLA mat varied with basis weights of nano-PLA membranes
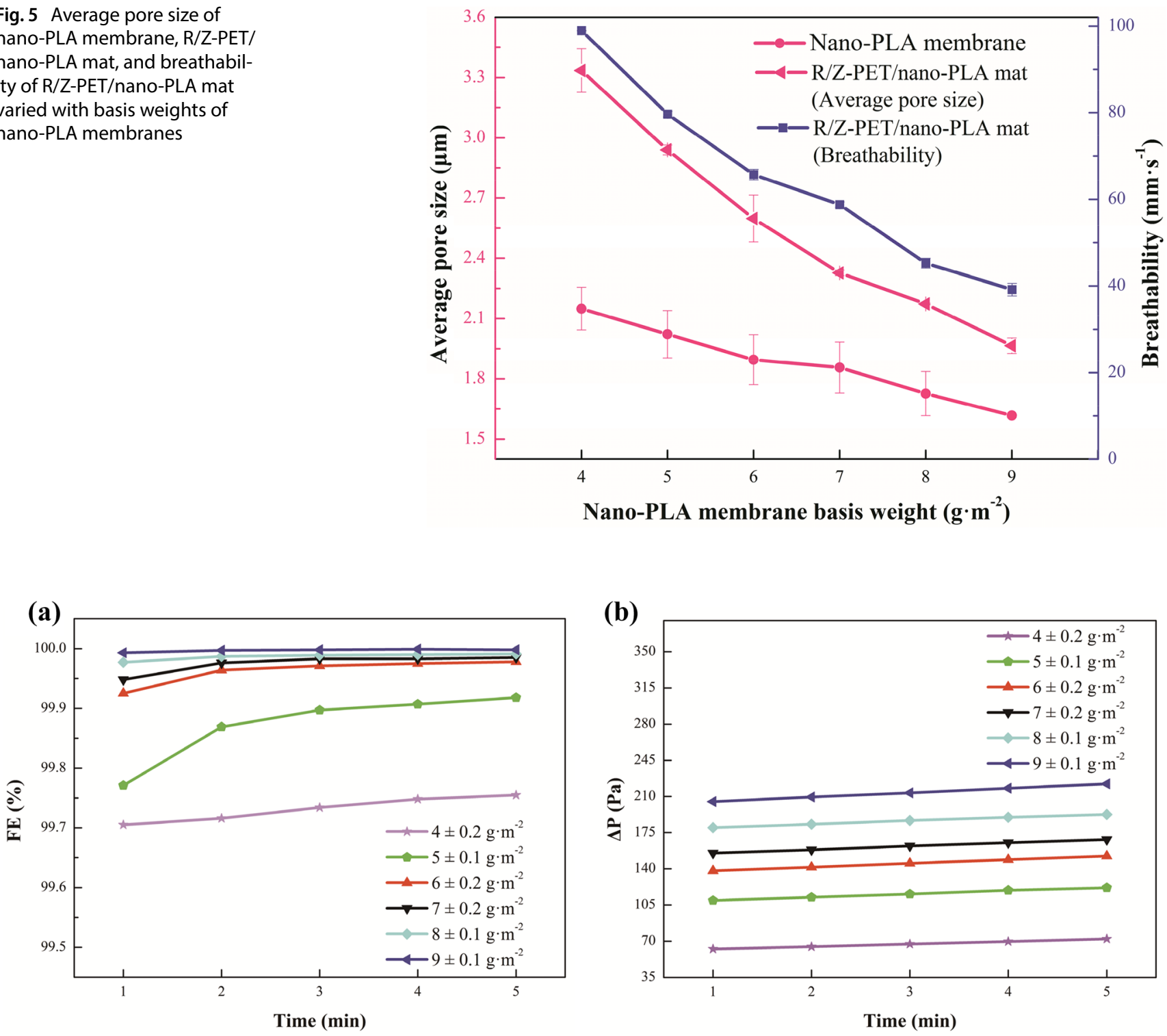

(b)

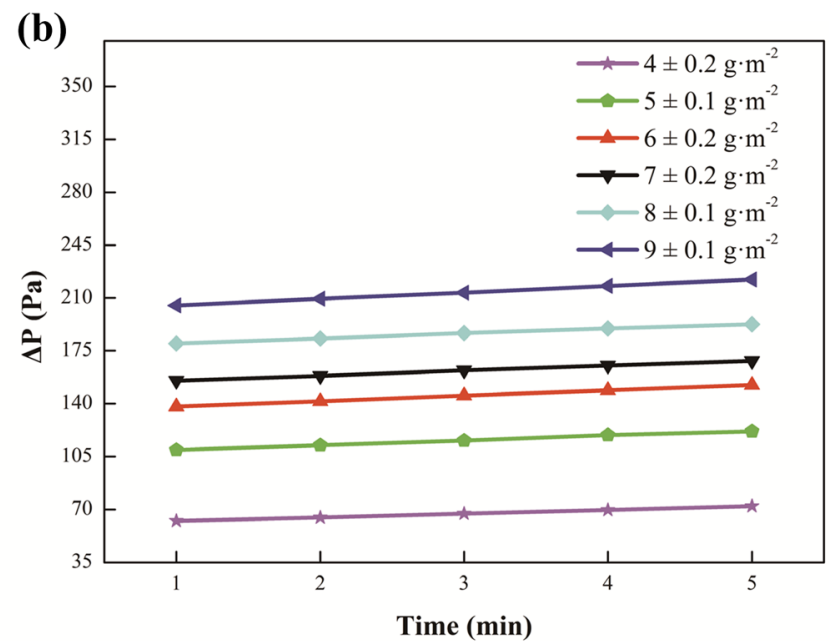

(c)

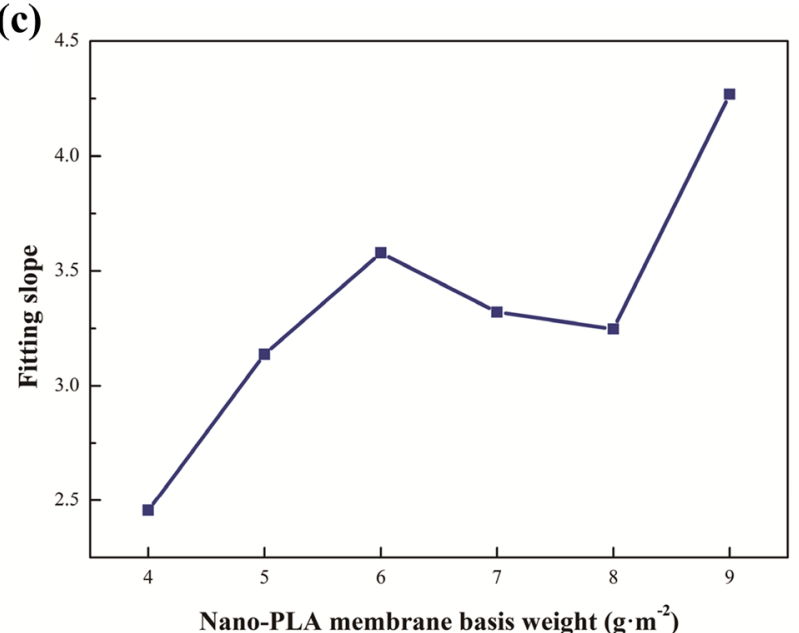

Fig. 6 a FE, $\mathbf{b} \Delta \mathrm{P}$ of R/Z-PET/nano-PLA mat under continuous loading time and $\mathbf{c}$ the slope tendency of the $\Delta \mathrm{P}$ varied with the basis weight of nano-PLA membranes

\section{SN Applied Sciences}


Additionally, from Fig. 7, we found that, as the basis weight of nano-PLA membrane increasing, particles intercepted in the surface of filtration material increase, and this is the results of the densifying of fibers in nano-PLA membrane and narrowing of pore size in membrane.

Aerosol particles can be classified as mono-dispersed and polydispersed samples according to particles size in filtration. The penetration capacity of particles is related to the nano-PLA membrane weight or thickness. Manipulation of this equation gives as follows [43],

$\ln p=-\alpha X$

where $P$ is the penetration of aerosol particles through the filtration material, $\alpha$ is the filtration index, and $X$ is the basis weight of nano-fiber membrane. The curve of penetration of aerosol versus basis weight of nano-PLA membrane conform to the polydisperse distribution of the testing $\mathrm{NaCl}$ particles (Fig. 8). The QF of thicker filtration materials decrease steadily when polydisperse aerosols are studied, thus the value of QF shows no big significant [44].

\subsection{Residual charge on nano-PLA membrane and its influence on filtration properties of R/Z-PET/ nano-PLA mat}

During electrospinning process, a high voltage electric field forms between the tip of needle and the collection layer, thus polarized charges resident on the surface of fiber, which cannot dissipate immediately. The residual charges enhance the temporary filtration ability $[45,46]$.

Tendency of charge dissipation depends on properties of fiber materials itself such as conductivity and hydrophobic or hydrophilic. For hydrophobic nano-PLA membrane, the surface voltage decreases from 2.9 to $0.35 \mathrm{kV}$ under an equilibrium condition for up to 145 days (Fig. 9a). The filtration performance of R/Z-PET/nano-PLA mat before and after electret elimination treatment (EET-R/Z-PET/ nano-PLA mat) is studied and results show the FE of EETR/Z-PET/nano-PLA mat drops from 99.992 to $99.985 \%$, but the $\Delta \mathrm{P}$ slightly increases from 201.11 to $205.14 \mathrm{~Pa}$ (Fig. 9b). This means the surface residual charge contributes some effect on the overall filtration performance of the R/Z-PET/ nano-PLA mat.

The QF $\left(0.043 \mathrm{~Pa}^{-1}\right)$ of EET-R/Z-PET/nano-PLA shows the highest value as compared with those of other R-PET/ nano-PLA mat (Table 1) except the R/Z-PET/nano-PLA mat. This further indicates that the excellent filtration performance of R/Z-PET/nano-PLA mat benefit from its zigzag curved structure of R-PET fiber.

\subsection{The dust-holding capacity of optimal R/Z-PET/ nano-PLA mat}

R/Z-PET/nano-PLA mats with different weights of nanoPLA membrane were prepared and their filtration properties were evaluated. The highest efficiency filtration material (>99.992\%) with a $\Delta \mathrm{P}$ of $201.11 \mathrm{~Pa}$ achieved.

Considering the basis requirement of filtration, we further investigated the dust-holding capacity of R/Z-PET/ nano-PLA mat under loading time of 5,15 and $30 \mathrm{~min}$, respectively (Fig. 10), and competed that of $R_{2.5}-\mathrm{PET} /$ nanoPLA mat under loading time of $30 \mathrm{~min}$.

Results show that the numbers of captured particles obvious increase on the surface of R/Z-PET/nano-PLA mat with prolonging loading time. The intercepted particles limit the pore size inside the filtration material, resulting in an improvement of FE of material itself. As a consequence, the dust-holding capacity is enhance
Fig. 7 SEM images of R/Z-PET/ nano-PLA fluffy filtration material under continue loading time
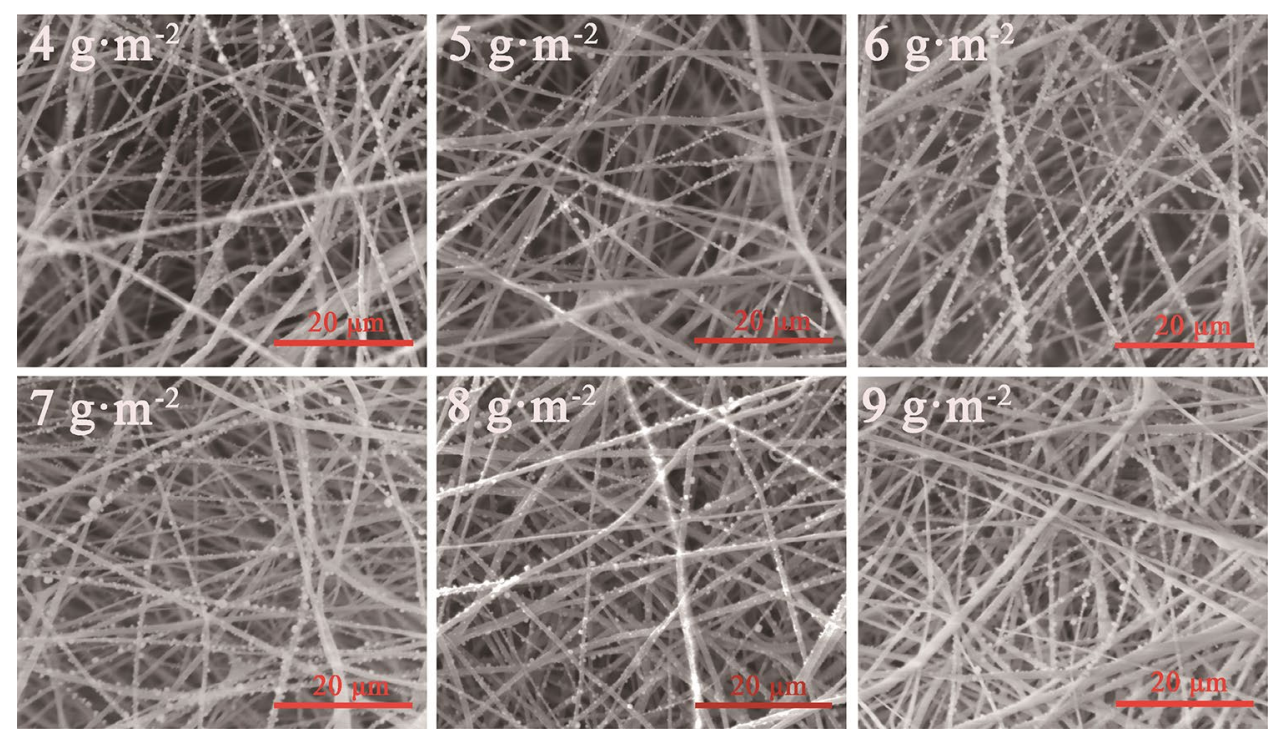
Fig. 8 Illustration of nano-PLA membrane weight efficiency of R/Z-PET/nano-PLA fluffy filtration material

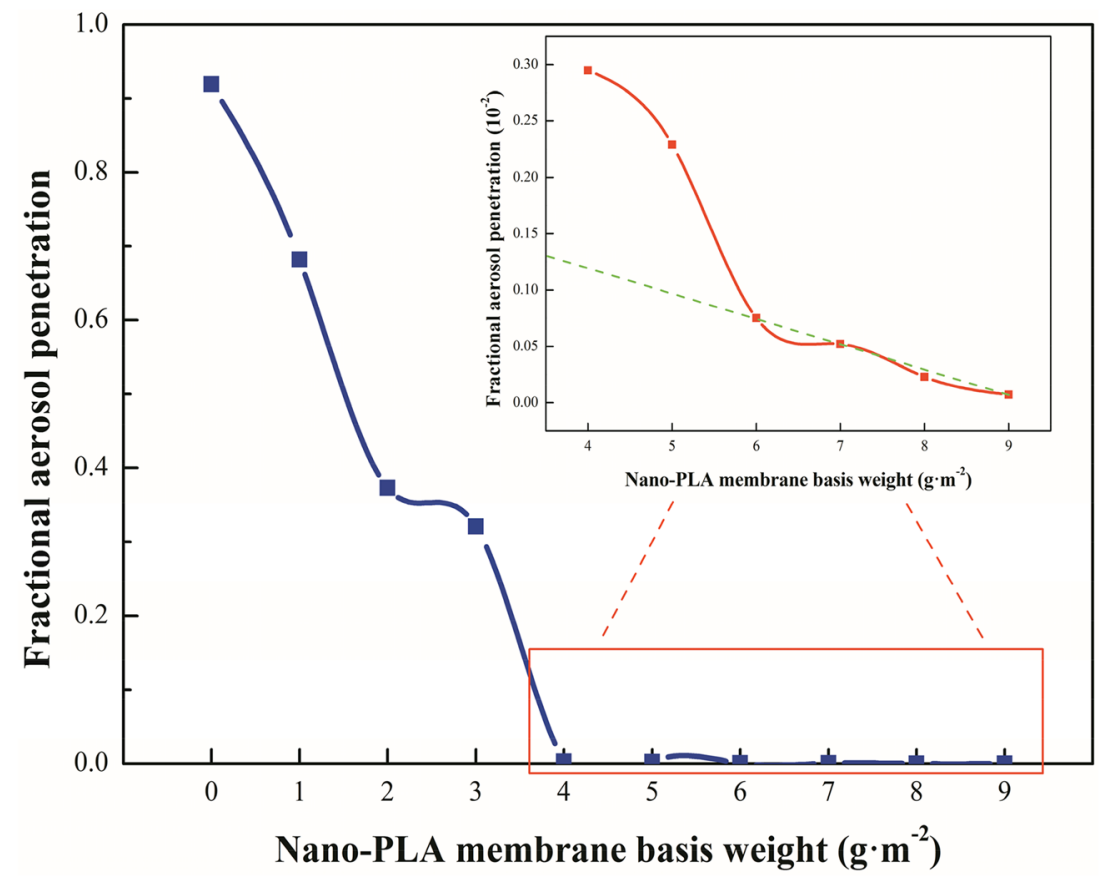

(a)

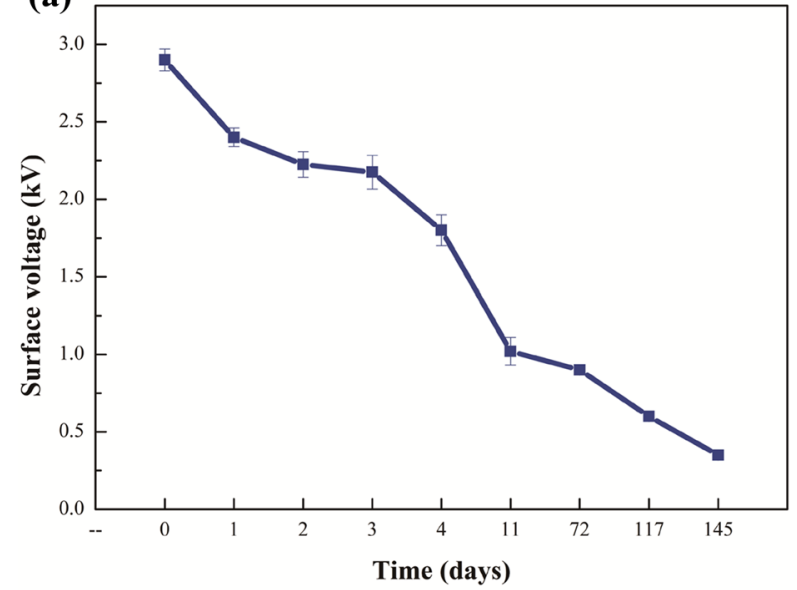

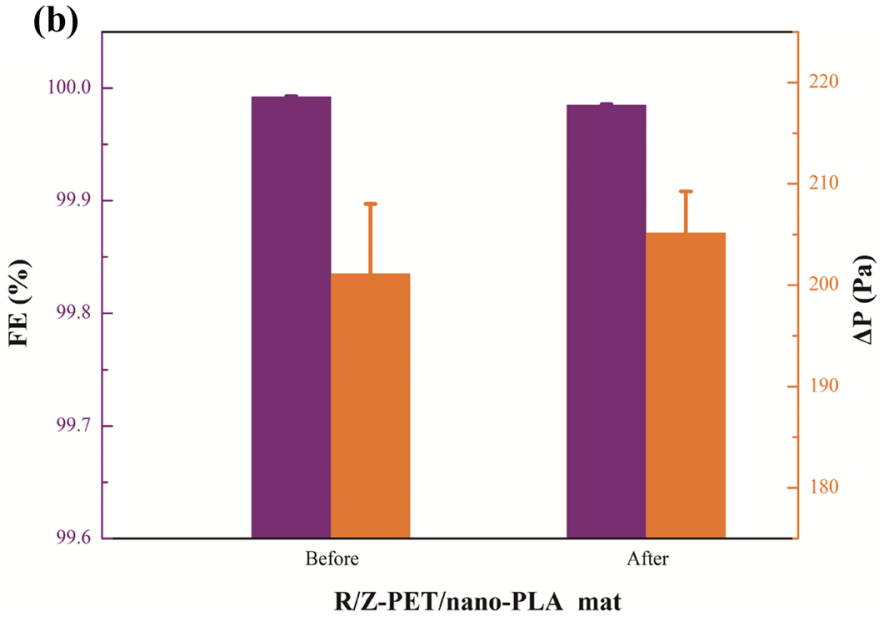

Fig. 9 Surface voltage decayed with storage time (a) and filtration performance before and after electret elimination treatment (b) of R/Z$\mathrm{PET} /$ nano-PLA mat (the basis weight of nano-PLA membrane is $9 \pm 0.1 \mathrm{~g} \mathrm{~m}^{-2}$ )

from 3.8 to 4.7 times. The dust-holding capacity of R/Z$\mathrm{PET} /$ nano-PLA mat $\left(1.0 \mathrm{~g} \mathrm{~m}^{-2}\right)$ was improved comparing with that of $R_{2.5} / \mathrm{N}-\mathrm{PET} /$ nano-PLA mat $\left(0.9 \mathrm{~g} \mathrm{~m}^{-2}\right)$ after loading for $30 \mathrm{~min}$. The fluffy $3 \mathrm{D}$ gradient structure in $\mathrm{R} / \mathrm{Z}-\mathrm{PET} /$ nano-PLA mat provides more space for the particles and extends the effective time for the particles diffusion inside the R/Z-PET layer [47], which results in the higher dust-holding capacity and FE (Fig. 4).

\section{SN Applied Sciences}

\subsection{Filtration mechanism of R-PET/nano-PLA mats}

For sub-micron aerosols, the filtration mechanism of filter primarily is a diffusion process. The particles leave the flowing streamline, move randomly in air, and are finally captured through the Brownian motion. Another capture mechanism is the interception for $100 \mathrm{~nm}$ and other larger aerosols [12, 48 , 49]. Figure 11 exhibits the schematic of airflow in R-PET 


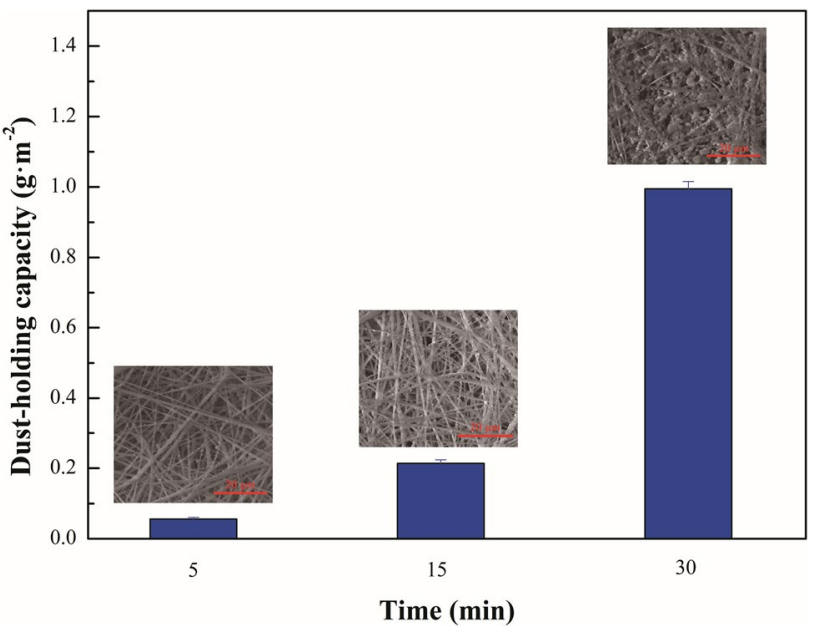

Fig. 10 SEM images and dust-holding capacity of R/Z-PET/nanoPLA mat under different loading time (the basis weight of nanoPLA membrane is $9 \pm 0.1 \mathrm{~g} \mathrm{~m}^{-2}$ )

substrate. For linear fiber (Fig. 11a), high percentage airflow is an even dent resistance instead of retarding flowing when the air run down meeting the surface of fiber. Thus, the filter often shows a high resistance.

For curved fiber, an interpenetrating micro/nanofibers gradient fiber layer could impose a pressure gradient that enhance the FE and reduce $\Delta \mathrm{P}$ of filtration material [20]. More significantly, the zigzag fiber (Fig. 11b) is beneficial to the "slip effect" of air on the surface of fiber. The computational equation of its effective functional surface (Fig. 11c) is as follows,

$d=L \cdot \cos \left(\frac{\pi-\theta}{2}\right)=L \cdot \sin \frac{\theta}{2}\left(0<\theta \leq 90^{\circ}\right)$ where $d$ is the effective functional surface of zigzag fiber, $L$ is the length of folding angle of zigzag fiber, and $\theta$ is folding angle of zigzag fiber. The effective functional surface of zigzag fiber shows the biggest value when the folding angle is at $90^{\circ}$. If the zigzag fiber orient at $45^{\circ}$ along the airflow direction (Fig. 11c), then half of fibers can posit at the same direction to the airflow, which means the friction of air on the surface of fiber will be at the minimum value. This tendency will not happen if the fiber curved in S shape. On the other hand, the stabilization of zigzag is more than that of the S-curved R-PET fiber. Though the S-shaped R-PET fiber (Fig. 11d) also has curved angle, this angle easily deforms [50] because of the unstable curved of fiber and transforms into linear shape that reduces the "slip effect" of airflow, and thus decreases the performance of filtration materials.

Figure 12 demonstrates the FE and $\triangle \mathrm{P}$ of nano-PLA membrane or R-PER web placed in windward. The growth rate of $\triangle \mathrm{P}$ of R/Z-PET/nano-PLA mat shows the slowest increase tendency as slop values in Table 2 . This is the result that half of air resistance on the surface of R/Z-PET fiber changes from dent resistance to slip resistance.

The R/Z-PET web in fluffy 3D R/Z-PET/nano-PLA mat provides more airflow reflecting effect, and then changes the airflow direction at the surface of zigzag fiber, which extends the retention time of airflow through the whole filtration material [51]. The Z-fiber and 3D gradient material create a "slip effect" of airflow, which promotes the penetration of airflow [39]. Furthermore, the zigzag fiber will improve functional surface of material for filtration function, which increases the chance of an inertial collision between the nanofiber and the airborne particles on a Brownian diffusion mechanism, resulting in an increased
Fig. 11 Schematic mechanism of airflows through linear (a), horizontally placed zigzag (b), zigzag fiber $45^{\circ}$ orienting along the air flow direction and the effective functional surface illustrated from zigzag (c) and horizontally placed S shape (d) fiber (a)

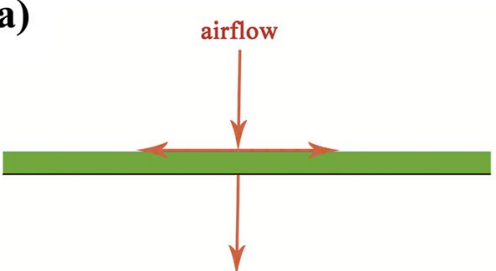

(c)

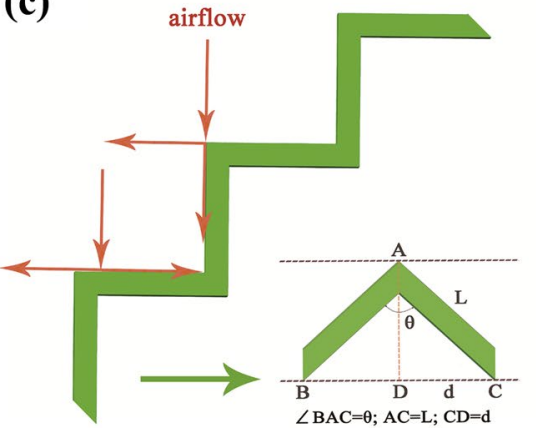

(b)

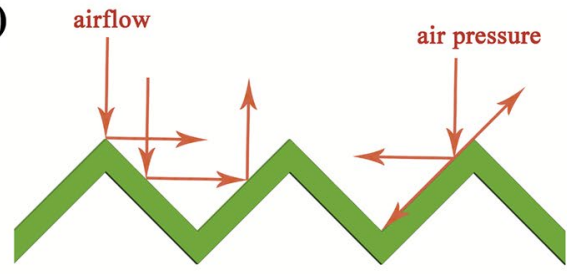

(d)

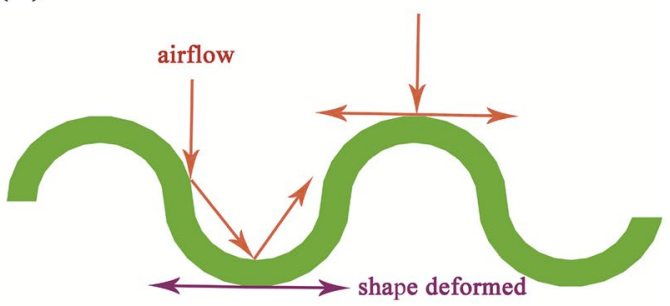




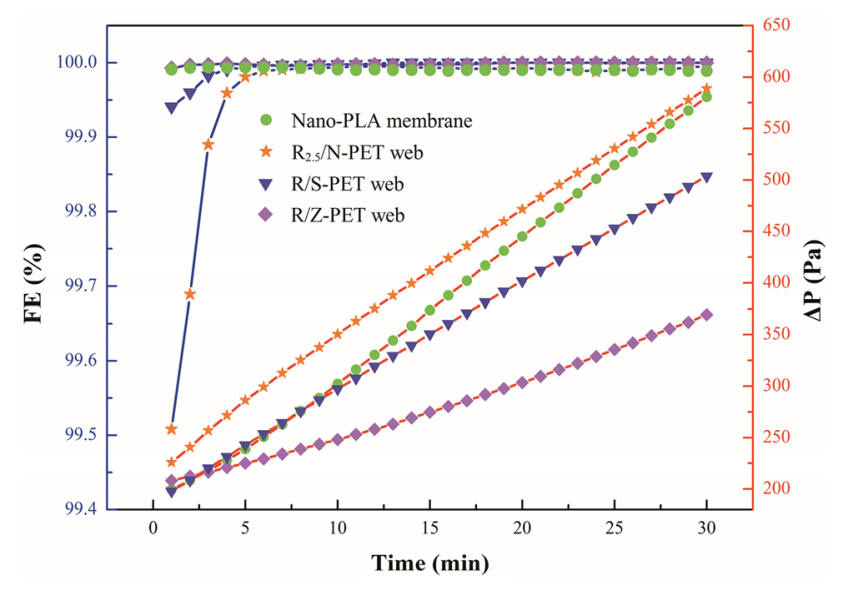

Fig. $12 \mathrm{FE}$ and $\Delta \mathrm{P}$ of different filtration mats charge with loading time, and the mats are placed in windward (the basis weight of nano-PLA membrane is $9 \pm 0.1 \mathrm{~g} \mathrm{~m}^{-2}$ )

Table 2 Fitting of $\Delta \mathrm{P}$ with different materials in windward (from Fig. 12)

\begin{tabular}{lrl}
\hline Materials & Slope & $\mathrm{R}^{2}$ \\
\hline Nano-PLA membrane & 13.61 & 0.9986 \\
$\mathrm{R}_{2.5}$ /N-PET web & 12.31 & 0.9989 \\
R/S-PET web & 10.51 & 0.9997 \\
R/Z-PET web & 5.57 & 0.9938 \\
\hline
\end{tabular}

interception probability of particles for filtration materials. FE of R/Z-PET/nano-PLA mat is up to $99.992 \%$ and the $\triangle \mathrm{P}$ is $201.11 \mathrm{~Pa}$. More significantly, the fluffy structure of $\mathrm{R} / \mathrm{Z}-\mathrm{PET}$ can extremely decrease the growth rate of $\triangle \mathrm{P}$. The environmental-friendly R/Z-PET/nano-PLA mats exhibit prospect in air pollution control applications.

\section{Conclusions}

Biodegradable PLA and post-used PET bottles were used as raw materials for electrospun nano-PLA fiber membrane and direct industrial produced R-PET fiber web. An efficient integrated 3D fluffy R/Z-PET/nano-PLA mats using for high efficacy air filtration material were designed to reduce $\triangle \mathrm{P}$. Optimal design of R/Z-PET/nano-PLA mat shows a high $\mathrm{FE}$ of $99.992 \%$, a low $\triangle \mathrm{P}$ of $201.11 \mathrm{~Pa}$, and the $Q F$ value of $0.047 \mathrm{~Pa}^{-1}$. The residual charges on the surface of nano-PLA membrane show some contributed to filtration performance of mats. The zigzag curved fluffy structure R-PET fibers with a folding angle of $90^{\circ}$ provide more chance for particle travelling inside the filtration material and decrease the friction between fiber and particles when the location angle of R-PET fiber is $45^{\circ}$ along the airflow direction. Otherwise, the fluffy structures significantly decrease the growth rate of $\Delta \mathrm{P}$ and enhance the dust-holding capacity of mat. Therefore, the environmental-friendly R/Z-PET/nano-PLA mat establishes a novel design of air filtration material as a powerful strategy would present in various applications ranging from industrial security to environmental governance.

Funding This study was funded by the National Natural Science Foundation of China-Youth Foundation (No. 5110620) and Guangdong Science and Technology Department (No. 2016A010103008).

\section{Compliance with ethical standards}

Conflict of interest The authors declare that they have no conflict of interest.

\section{References}

1. Thavasi V, Singh G, Ramakrishna S (2008) Electrospun nanofibers in energy and environmental applications. Energy Environ Sci 1(2):205-221

2. Lee ES, Fung CCD, Zhu Y (2015) Evaluation of a high efficiency cabin air (HECA) filtration system for reducing particulate pollutants inside school buses. Environ Sci Technol 49(6):3358-3365

3. Zhang S, Tang N, Cao L et al (2016) Highly integrated polysulfone/polyacrylonitrile/polyamide- 6 air filter for multilevel physical sieving airborne particles. ACS Appl Mater Interfaces 8(42):29062-29072

4. Wan H, Wang N, Yang J et al (2014) Hierarchically structured polysulfone/titania fibrous membranes with enhanced air filtration performance. J Colloid Interface Sci 417(3):18-26

5. Liu C, Hsu PC, Lee HW et al (2015) Transparent air filter for highefficiency $\mathrm{PM}_{2.5}$ capture. Nat Commun 6:1-9

6. Brochocka A, Majchrzycka K, Makowski K (2013) Modified meltblown nonwovens for respiratory protective devices against nanoparticles. Fibres Text East Eur 100(4):106-111

7. Kilic A, Shim E, Pourdeyhimi B (2015) Electrostatic capture efficiency enhancement of polypropylene electret filters with barium titanate. Aerosol Sci Technol 49(8):666-673

8. Cai RR, Zhang LZ, Bao AB (2018) PM collection performance of electret filters electrospun with different dielectric materialsa numerical modeling and experimental study. Build Environ 131:210-219

9. Thakur R, Das D, Das A (2014) Study of charge decay in coronacharged fibrous electrets. Fibers Polym 15(7):1436-1443

10. Al-Attabi R, Dumée LF, Kong L et al (2018) High efficiency poly(acrylonitrile) electrospun nanofiber membranes for airborne nanomaterials filtration. Adv Eng Mater 20(1):1-10

11. Wang N, Yang Y, Al-Deyab SS et al (2015) Ultra-light 3D nanofibre-nets binary structured nylon 6-polyacrylonitrile membranes for efficient filtration of fine particulate matter. J Mater Chem A 3(47):23946-23954

12. Zhang S, Shim WS, Kim J (2009) Design of ultra-fine nonwovens via electrospinning of nylon 6: spinning parameters and filtration efficiency. Mater Des 30(9):3659-3666

13. Liu B, Zhang S, Wang X et al (2015) Efficient and reusable polyamide-56 nanofiber/nets membrane with bimodal structures for air filtration. J Colloid Interface Sci 457:203-211

14. Gao H, Yang Y, Akampumuza $\mathrm{O}$ et al (2017) A low filtration resistance three-dimensional composite membrane fabricated via 
free surface electrospinning for effective $\mathrm{PM}_{2.5}$ capture. Environ Sci Nano 4(4):864-875

15. Balgis R, Murata H, Goi Y et al (2017) Synthesis of dual-size cellulose-polyvinylpyrrolidone nanofiber composites via one-step electrospinning method for high-performance air filter. Langmuir 33(24):6127-6234

16. Zhang Q, Welch J, Park $\mathrm{H}$ et al (2010) Improvement in nanofiber filtration by multiple thin layers of nanofiber mats. J Aerosol Sci 41(2):230-236

17. Zhang $Y$, Yuan S, Feng $X$ et al (2016) Preparation of nanofibrous metal-organic framework filters for efficient air pollution control. J Am Chem Soc 138(18):5785-5788

18. Jackiewicz-Zagórska A, Szwast M, Gac J et al (2018) New methods of natural gas adjusting for technological purposes based on modern filtration materials. Ecol Chem Eng S 25(1):61-72

19. Su J, Yang $G$, Cheng $C$ et al (2017) Hierarchically structured $\mathrm{TiO}_{2} /$ PAN nanofibrous membranes for high-efficiency air filtration and toluene degradation. J Colloid Interface Sci 507:386-396

20. Zhu M, Han J, Wang F et al (2017) Electrospun nanofibers membranes for effective air filtration. Macromol Mater Eng 302(1):1-27

21. Cheng Z, Zhang Y, Han Z et al (2016) A novel preparation of anti-layered poly(vinylalcohol)-polyacrylonitrile (PVA/PAN) membrane for air filtration by electrospinning. RSC Adv 6(88):85545-85550

22. Zhang S, Liu H, Yin X et al (2016) Anti-deformed polyacrylonitrile/polysulfone composite membrane with binary structures for effective air filtration. ACS Appl Mater Interfaces 8(12):8086-8095

23. Zhao X, Li Y, Hua T et al (2017) Cleanable air filter transferring moisture and effectively capturing $\mathrm{PM}_{25}$. Small 13(11):1-11

24. Patanaik A, Jacobs V, Anandjiwala RD (2010) Performance evaluation of electrospun nanofibrous membrane. J Memb Sci 352(1-2):136-142

25. Zulfi A, Munir MM, Hapidin DA et al (2018) Air filtration media from electrospun waste high-impact polystyrene fiber membrane. Mater Res Express 5(3):035049

26. Liu X, Souzandeh H, Zheng Y et al (2017) Soy protein isolate/ bacterial cellulose composite membranes for high efficiency particulate air filtration. Compos Sci Technol 138:124-133

27. Wang Z, Yan F, Pei H et al (2018) Antibacterial and environmentally friendly chitosan/polyvinyl alcohol blend membranes for air filtration. Carbohydr Polym 198:241-248

28. Strain IN, Wu Q, Pourrahimi AM et al (2015) Electrospinning of recycled $P E T$ to generate tough mesomorphic fibre membranes for smoke filtration. J Mater Chem A 3(4):1632-1640

29. Chang G, Zhu X, Li A et al (2016) Formation and self-assembly of 3D nanofibrous networks based on oppositely charged jets. Mater Des 97:126-130

30. Kim HL, Lee JH, Seo HJ et al (2014) Fabrication of threedimensional poly(lactic-co-glycolic acid) mesh by electrospinning using different solvents with dry ice. Macromol Res 22(4):377-381

31. Casasola R, Thomas NL, Trybala A, Georgiadou S (2014) Electrospun poly lactic acid (PLA) fibres: effect of different solvent systems on fibre morphology and diameter. Polymer 55(18):4728-4737

32. Cheng W, Yu Q, Qiu Z, Yan Y (2013) Effects of different ionic liquids on the electrospinning of a polyacrylonitrile polymer solution. J Appl Polym Sci 130(4):2359-2368

33. Sugden $S$ (1925) The determination of surface tension from the rise in capillary tubes. J Am Chem Soc 47(1):60-64
34. Mazinani S, Ajji A, Dubois C (2009) Morphology, structure and properties of conductive PS/CNT nanocomposite electrospun mat. Polymer 50(14):3329-3342

35. Koski A, Yim K, Shivkumar S (2004) Effect of molecular weight on fibrous PVA produced by electrospinning. Mater Lett 58(3):493-497

36. Amini N, Kalaee M, Mazinani S et al (2013) Morphological optimization of electrospun polyacrylamide/MWCNTs nanocomposite nanofibers using Taguchi's experimental design. Int J Adv Manuf Technol 69(1-4):139-146

37. Lou LH, Qin XH, Zhang H (2017) Preparation and study of lowresistance polyacrylonitrile nano membranes for gas filtration. Text Res J 87(2):208-215

38. Li L, Frey M, Green T (2006) Modification of air filter media with nylon-6 nanofibers. J Eng Fiber Fabr 1(1):1-22

39. Wang N, Si Y, Wang N et al (2014) Multilevel structured polyacrylonitrile/silica nanofibrous membranes for high-performance air filtration. Sep Purif Technol 126(15):44-51

40. Shou D, Ye L, Fan J (2014) Gas transport properties of electrospun polymer nanofibers. Polymer 55(14):3149-3155

41. Solanas C, Herrero S, Dasari A et al (2014) Insights into the production and characterization of electrospun fibers from regenerated silk fibroin. Eur Polym J 60(60):123-134

42. Chen CC, Chen WY, Huang SH et al (2001) Experimental study on the loading characteristics of needlefelt filters with micrometersized monodisperse aerosols. Aerosol Sci Technol 34:262-273

43. Brown RC (1993) Air filtration: an integrated approach to the theory and applications of fibrous filters. Pergamon Press, Oxford, p 5

44. Brown RC (1993) Air filtration: an integrated approach to the theory and applications of fibrous filters. Pergamon Press, Oxford, p 272

45. Li X, Wang N, Fan G et al (2015) Electreted polyetherimide-silica fibrous membranes for enhanced filtration of fine particles. $J$ Colloid Interface Sci 439:12-20

46. Cho BM, Nam YS, Cheon JY, Park WH (2014) Residual charge and filtration efficiency of polycarbonate fibrous membranes prepared by electrospinning. J Appl Polym Sci 132(1):1-7

47. Liu J, Zhang $X$, Zhang $\mathrm{H}$ et al (2017) Low resistance bicomponent spunbond materials for fresh air filtration with ultra-high dust holding capacity. RSC Adv 7:43879-43887

48. Barhate RS, Ramakrishna S (2007) Nanofibrous filtering media: filtration problems and solutions from tiny materials. J Memb Sci 296(1):1-8

49. Leung WWF, Choy HF (2018) Transition from depth-to-surface filtration for a high-efficiency, high-skin effect, nanofiber filter under continuous nano-aerosol loading. Chem Eng Sci 182:67-76

50. Wang N, Zhang Z, Yue F, Zhang X (2019) Design and analysis of translational joints using corrugated flexural beams with conic curve segments. Mech Mach Theory 132:223-235

51. Yang Y, Zhang S, Zhao X et al (2015) Sandwich structured polyamide-6/polyacrylonitrile nanonets/bead-on-string composite membrane for effective air filtration. Sep Purif Technol $152: 14-22$

Publisher's Note Springer Nature remains neutral with regard to jurisdictional claims in published maps and institutional affiliations. 